PROCEEDINGS OF THE

AMERICAN MATHEMATICAL SOCIETY

Volume 136, Number 7, July 2008, Pages 2631-2637

S 0002-9939(08)09443-4

Article electronically published on February 29, 2008

\title{
WORD LENGTH IN SURFACE GROUPS WITH CHARACTERISTIC GENERATING SETS
}

\author{
DANNY CALEGARI
}

(Communicated by Daniel Ruberman)

\begin{abstract}
A subset of a group is characteristic if it is invariant under every automorphism of the group. We study word length in fundamental groups of closed hyperbolic surfaces with respect to characteristic generating sets consisting of a finite union of orbits of the automorphism group, and show that the translation length of any element with a nonzero crossing number is positive, and bounded below by a constant depending only (and explicitly) on a bound on the crossing numbers of generating elements. This answers a question of Benson Farb.
\end{abstract}

\section{INTRODUCTION}

Let $S$ be a closed, orientable surface of genus at least 2. Then $S$ admits a (nonunique) hyperbolic structure. Let $a \in \pi_{1}(S)$ and let $[a]$ denote the conjugacy class of $a$. Once we have fixed a hyperbolic structure on $S$, each nontrivial conjugacy class $[a]$ determines a unique closed (unparameterized) geodesic $\gamma(a)$ in the corresponding free homotopy class on $S$.

Note that $\gamma(a)$ depends only on the conjugacy class of $a$, so we could write $\gamma([a])$, but we choose the notation $\gamma(a)$ for simplicity.

Definition 1.1. If $a$ is primitive, a self-intersection of $\gamma(a)$ is an unordered pair of geodesics $\tilde{\gamma}_{1}, \tilde{\gamma}_{2}$ in the universal cover of $S$ which cover $\gamma(a)$ and have linked endpoints in the circle at infinity, up to the action of the deck group $\pi_{1}(S)$ on such pairs.

The crossing number of $a$, denoted $\operatorname{cr}(a)$, is the number of self-intersections of $\gamma(a)$. If $a=b^{n}$, then set $\operatorname{cr}(a)=n^{2} \operatorname{cr}(b)$.

Since $\gamma(a)$ depends only on the conjugacy class of $a$, it follows that cr also depends only on the conjugacy class of $a$.

Moreover, since linking data does not depend on the choice of a hyperbolic structure on $S$ (although the configuration of $\gamma(a)$ in $S$ typically will), it follows that $\operatorname{cr}(a)$ does not depend on the choice of a hyperbolic structure on $S$.

Lemma 1.2. The crossing number is constant on orbits of $\operatorname{Out}\left(\pi_{1}(S)\right)$.

Received by the editors May 21, 2007.

2000 Mathematics Subject Classification. Primary 57M07.

(C)2008 American Mathematical Society Reverts to public domain 28 years from publication 
Proof. Choose a hyperbolic structure $g$ on $S$, and let $[\phi]$ be an element of the mapping class group $\operatorname{MCG}(S)$, which is equal to Out $\left(\pi_{1}(S)\right)$ by Dehn-Nielsen, represented by a diffeomorphism $\phi: S \rightarrow S$. If $[b]=[\phi]([a])$, then $\gamma(b)$ in the $\left(\phi^{-1}\right)^{*} g$ metric is the image of $\gamma(a)$ in the $g$ metric under the diffeomorphism $\phi$.

For each nonnegative integer $n$, let $S_{n}$ be the subset consisting of all elements with a crossing number at most $n$, and let $S_{n}^{\prime}$ be the subset of $S_{n}$ consisting of primitive elements.

For each $a \in \pi_{1}(S)$ define $w_{n}(a)$ to be the word length of $a$ with respect to the generating set $S_{n}$ and $w_{n}^{\prime}(a)$ to be the word length of $a$ with respect to the generating set $S_{n}^{\prime}$. Obviously $w_{n}^{\prime}(a) \geq w_{n}(a)$ for any $n$, since $S_{n}^{\prime} \subset S_{n}$.

Remark 1.3. There is an equality $\operatorname{cr}(b)=\operatorname{cr}\left(b^{-1}\right)$ for every $b \in \pi_{1}(S)$, so the $S_{n}$ and $S_{n}^{\prime}$ are symmetric generating sets.

Remark 1.4. Each $S_{n}^{\prime}$ is a finite union of primitive $\operatorname{Aut}\left(\pi_{1}(S)\right)$ orbits. Conversely, every finite union of primitive Aut $\left(\pi_{1}(S)\right)$ orbits is contained in $S_{n}^{\prime}$ for some $n$, and every finite union of (not necessarily primitive) Aut $\left(\pi_{1}(S)\right)$ orbits is contained in $S_{n}$ for some $n$.

Benson Farb asked whether the diameter of $\pi_{1}(S)$ with respect to $S_{0}$ is infinite, and in general to understand the geometry of the Cayley graph of $\pi_{1}(S)$ with respect to $\operatorname{Aut}\left(\pi_{1}(S)\right)$-invariant generating sets. The purpose of this note is to prove the following theorem:

Theorem A. There are constants $C_{1}(S), C_{2}(S), C_{3}(S)$ such that for any nonnegative integers $n, m$ and any $a \in \pi_{1}(S)$ with $\operatorname{cr}(a)>0$, there is an inequality

$$
w_{n}\left(a^{m}\right) \geq \frac{C_{1} m}{\sqrt{n}+C_{2}}-C_{3} .
$$

Notice that the constants $C_{1}, C_{2}, C_{3}$ do not depend on $n$ or on the choice of $a \in \pi_{1}(S)$.

Remark 1.5. The dependence of the constants $C_{i}$ on the surface $S$ is also probably unnecessary.

Question 1.6. Let $a \in \pi_{1}(S)$ have $\operatorname{cr}(a)=0$. What is the growth rate of the word length of $a^{m}$ with respect to the generating set $S_{n}^{\prime}$ ?

\section{Counting Quasimorphisms}

2.1. Quasimorphisms. The usual way to obtain lower bounds on word length is to find a suitable function which is bounded on a generating set, which grows linearly on powers, and which is almost subadditive under multiplication. A rich source of such functions is quasimorphisms.

Definition 2.1. Let $G$ be a group. A quasimorphism is a function $\phi: G \rightarrow \mathbb{R}$ for which there is a smallest real number $D(\phi) \geq 0$ called the defect, with the property that for all $a, b \in G$ there is an inequality

$$
|\phi(a)+\phi(b)-\phi(a b)| \leq D(\phi) .
$$

A quasimorphism is homogeneous if $\phi\left(a^{n}\right)=n \phi(a)$ for all integers $n$. 
If $\phi$ is a quasimorphism, the function

$$
\bar{\phi}(a):=\lim _{n \rightarrow \infty} \frac{\phi\left(a^{n}\right)}{n}
$$

is a homogeneous quasimorphism, whose defect satisfies $D(\bar{\phi}) \leq 2 D(\phi)$. Homogeneous quasimorphisms are class functions. See e.g. [1] or 4] for details.

2.2. Hyperbolic groups. We assume the reader is familiar with the basic properties of hyperbolic geometry, hyperbolic groups and $\delta$-hyperbolic spaces, quasigeodesics, Morse Lemma, convexity of distance function, etc. For a reference, see Bridson-Haefliger [2] or Gromov [7].

Let $G$ be a group which is $\delta$-hyperbolic with respect to a generating set $A$. Epstein-Fujiwara [6], generalizing a construction due to Brooks [3], define so-called counting quasimorphisms as follows.

Definition 2.2. Let $\sigma$ be an oriented simplicial path in the Cayley graph $C_{A}(G)$, and let $\sigma^{-1}$ denote the same path with the opposite orientation. A copy of $\sigma$ is a translate $a \cdot \sigma$ with $a \in G$. For $\alpha$ an oriented simplicial path in $C_{A}(G)$, let $|\alpha|_{\sigma}$ denote the maximal number of disjoint copies of $\sigma$ contained in $\alpha$. For $a \in G$, define

$$
c_{\sigma}(a)=d(\mathrm{id}, a)-\inf _{\alpha}\left(\operatorname{length}(\alpha)-|\alpha|_{\sigma}\right)
$$

where the infimum is taken over all directed paths $\alpha$ in $C_{A}(G)$ from id to $a$.

Define a counting quasimorphism to be a function of the form

$$
h_{\sigma}(a):=c_{\sigma}(a)-c_{\sigma^{-1}}(a) .
$$

In the sequel we always assume that the length of $\sigma$ is at least 2. It is clear from this definition that the homogenization $\bar{h}$ of $h_{\sigma}$ is a class function. A path $\alpha$ as above which achieves the infimum for a given $\sigma$ and $a \in G$ is called a realizing path for $c_{\sigma}$. Since the length of any path is a nonnegative integer, a realizing path must exist for any $a$ and any $\sigma$.

Realizing paths have the following universal property:

Lemma 2.3 (Epstein-Fujiwara, Prop. 2.2 of [6]). Any realizing path for $c_{\sigma}$ is a $(K, \epsilon)$-quasigeodesic, where

$$
K=\frac{\operatorname{length}(\sigma)}{\operatorname{length}(\sigma)-1}, \quad \epsilon=\frac{2 \cdot \operatorname{length}(\sigma)}{\operatorname{length}(\sigma)-1} .
$$

Notice by our hypothesis that length $(\sigma)$ is at least 2 that $K \leq 2$ and $\epsilon \leq 4$. By the Morse Lemma there is a constant $C(\delta)$ such that every realizing path for $c_{\sigma}$ must be contained in the $C$-neighborhood of any geodesic from id to $a$. In particular, one obtains the following lemma:

Lemma 2.4. There is a constant $C(\delta)$ such that for any path $\sigma \in C_{S}(G)$ of length at least 2, and for any $a \in G$, if the $C$-neighborhood of any geodesic from id to a does not contain a copy of $\sigma$, then $c_{\sigma}(a)=0$.

Finally, the defect of $h_{\sigma}$ (and therefore of $\bar{h}_{\sigma}$ ) can be controlled independently of $\sigma$ :

Lemma 2.5 (Epstein-Fujiwara, Prop. 2.13 of [6]). Let $\sigma$ be a path in $C_{S}(G)$ of length at least 2 . Then there is a constant $C(\delta)$ such that $D\left(h_{\sigma}\right) \leq C$. 


\section{Stability of CROSSINGS}

If two geodesics in the hyperbolic plane intersect with a small angle, then as the geodesics are moved around slightly, the point of intersection might move wildly. Nevertheless, two geodesics which cross are close only in a compact region; for a small perturbation, the point of intersection cannot move outside that compact region.

Let $\alpha, \beta$ be two complete geodesics in the hyperbolic plane which cross transversely at $p$. Fix a small $\epsilon>0$. Let $\alpha_{\epsilon}$ denote the subset of $\alpha$ which intersects the $2 \epsilon$-neighborhood of $\beta$, and similarly define $\beta_{\epsilon}$. Let $\alpha^{\prime}, \beta^{\prime}$ be two geodesics which contain segments $\alpha_{\epsilon}^{\prime}, \beta_{\epsilon}^{\prime}$ which are $\epsilon$-close to $\alpha_{\epsilon}, \beta_{\epsilon}$, respectively. Then $\alpha^{\prime}, \beta^{\prime}$ have a transverse intersection which is contained in $\alpha_{\epsilon}^{\prime} \cap \beta_{\epsilon}^{\prime}$. We call such a pair of segments $\alpha_{\epsilon}, \beta_{\epsilon}$ an $\epsilon$-trap for the intersection $p$. Or, if $\epsilon$ is understood, just a trap for $p$.

Now let $S$ be a closed hyperbolic surface, and let $\gamma \subset S$ be a closed geodesic. Suppose $\alpha, \beta$ project to $\gamma$ by the covering projection.

Lemma 3.1. Let $\alpha, \beta, \gamma, S$ be as above, and let $\alpha_{\epsilon}, \beta_{\epsilon}$ be an $\epsilon$-trap for $p$, which projects to a self-intersection of $\gamma$. Suppose $8 \epsilon$ is less than the length of the shortest nontrivial curve on $S$. Then length $\left(\alpha_{\epsilon}\right)<\operatorname{length}(\gamma)+4 \epsilon$, and similarly for $\beta_{\epsilon}$.

Proof. By the definition of an $\epsilon$-trap, the endpoints of $\alpha_{\epsilon}$ and $\beta_{\epsilon}$ are $2 \epsilon$ apart. By the triangle inequality, there is an estimate

$$
\mid \text { length }\left(\alpha_{\epsilon}\right)-\operatorname{length}\left(\beta_{\epsilon}\right) \mid<4 \epsilon \text {. }
$$

$\operatorname{Suppose} \operatorname{length}\left(\alpha_{\epsilon}\right) \geq \operatorname{length}(\gamma)+4 \epsilon$ and therefore length $\left(\beta_{\epsilon}\right) \geq \operatorname{length}(\gamma)$. By hypothesis there are elements $a, b \in \pi_{1}(S)$ in the conjugacy class of $\gamma$ which stabilize $\alpha, \beta$, respectively, and act as translations through a distance length $(\gamma)$. Let $q$ be an endpoint of $\alpha_{\epsilon}$. Then $d\left(b^{-1} a(q), q\right) \leq 8 \epsilon$. But by hypothesis, this is shorter than the length of the shortest nontrivial curve on $S$, so $b=a$ and $\beta=\alpha$, contrary to hypothesis. It follows that length $\left(\alpha_{\epsilon}\right)<\operatorname{length}(\gamma)+4 \epsilon$, and the lemma is proved.

Lemma 3.2. Let $S, \epsilon$ be as above. Let $\gamma$ be a closed geodesic on $S$ with a transverse self-intersection. Let $\alpha$ be a geodesic in the hyperbolic plane covering $\gamma$, and let $\sigma$ be a geodesic segment in the hyperbolic plane which is $\epsilon$-close to a segment of $\alpha$, and satisfies length $(\sigma)>2 \cdot \operatorname{length}(\gamma)+4 \epsilon$. Then the projection of $\sigma$ to $S$ has a transverse self-intersection.

Proof. Let $\beta$ be another geodesic in the hyperbolic plane covering $\gamma$ such that $\alpha \cap \beta$ contains a point $p$ projecting to a transverse self-intersection of $\gamma$, and let $\alpha_{\epsilon}, \beta_{\epsilon}$ be an $\epsilon$-trap for $p$. By Lemma 3.1, length $\left(\alpha_{\epsilon}\right)+\operatorname{length}(\gamma) \leq \operatorname{length}(\sigma)$, so there is a translate of $\sigma$ by an element of the deck group which contains a segment $\alpha_{\epsilon}^{\prime}$ which is $\epsilon$-close to $\alpha_{\epsilon}$. Similarly, there is a translate of $\sigma$ containing a segment $\beta_{\epsilon}^{\prime}$ which is $\epsilon$-close to $\beta_{\epsilon}$. Since $\alpha_{\epsilon}, \beta_{\epsilon}$ is an $\epsilon$-trap for $p$, the segments $\alpha_{\epsilon}^{\prime}, \beta_{\epsilon}^{\prime}$ intersect transversely. The projections of $\alpha_{\epsilon}^{\prime}$ and $\beta_{\epsilon}^{\prime}$ are both contained in the projection of $\sigma$, and therefore this projection contains a point of self-intersection, as claimed.

Remark 3.3. Chris Leininger has observed that for every nonsimple primitive homotopy class of loop on a surface $S$, there is some hyperbolic structure on $S$ for which the geodesic representative intersects itself at a definite angle. This observation, together with the fact that $w_{n}$ is characteristic, could be used in place of the results in this section in the proof of Theorem A. 


\section{Proof of Theorem A}

We now give the proof of Theorem A.

Proof. Fix a hyperbolic structure on $S$. Fix a generating set $A$ for $\pi_{1}(S)$, and let $\delta$ be such that the Cayley graph with respect to this generating set is $\delta$-hyperbolic. Let $K, \epsilon^{\prime}$ be such that the Cayley graph $C_{A}\left(\pi_{1}(S)\right)$ is $\left(K, \epsilon^{\prime}\right)$ quasi-isometric to the hyperbolic plane (identified with the universal cover of $S$ ) by a fixed equivariant quasi-isometry. Note that $K, \epsilon^{\prime}$ can be chosen to depend only on $S$.

Let $a \in \pi_{1}(S)$ have $\operatorname{cr}(a)>0$. There is a constant $C_{1}(\delta,|A|)$ depending only on $\delta$ and the cardinality $|A|$ such that $a^{C_{1}}$ has an axis in $C_{A}\left(\pi_{1}(S)\right)$. We replace $a$ by a suitable conjugate $b$ of $a^{C_{1}}$ whose axis contains id. Note that $\gamma(b)$ is just a $C_{1}$-multiple of $\gamma(a)$, and therefore contains $C_{1}^{2} \operatorname{cr}(a)$ transverse self-intersections. Since $C_{1}$ depends only on $\delta,|A|$, and therefore only on $S$, it suffices to prove the theorem with $b$ in place of $a$.

Let $l \subset C_{A}\left(\pi_{1}(S)\right)$ be the (oriented) axis of $b$, which by hypothesis passes through id. Let $N$ be a sufficiently large number (we will say how large in a moment), and let $\sigma$ be the oriented segment of $l$ from id to $b^{N}$. Define the quasimorphism $h_{\sigma}$ as in Definition 2.2.

Claim 1 of Theorem $\mathrm{A}^{\prime}$ in 5 says that if the translation length of $b^{N}$ is sufficiently long (depending only on $\delta$ and $|A|$ ), then $c_{\sigma^{-1}}\left(b^{N m}\right)=0$ for all positive $m$. From the definition, $c_{\sigma}\left(b^{N m}\right)=m$ for all positive $m$, and therefore we have an equality $h_{\sigma}\left(b^{N m}\right)=m$, valid (by symmetry) for all integers $m$. We can make the translation length of $b^{N}$ long enough by replacing $b$ by a proper power if necessary, absorbing the constant (multiplicatively) into $C_{1}$ as above.

On the other hand, let $e \in S_{n}$. Observe that $e^{C_{1}}$ has an axis $l_{e}$. Note that $\operatorname{cr}\left(e^{C_{1}}\right) \leq C_{1}^{2} n$ by the definition of $S_{n}$. How many copies of $\sigma$ can there be in a realizing path for $c_{\sigma}$ on $e^{C_{1}}$ ? Each copy of $\sigma$ in such a realizing path defines a segment in $l_{e}$ which is in the $C_{2}$-neighborhood of a translate of $l$, where $C_{2}$ is as in Lemma 2.4

It follows that there is a constant $C_{3}$, depending only on $C_{2}, K, \epsilon^{\prime}$, such that suitable geodesics $\tilde{\gamma}(e)$ and $\tilde{\gamma}(b)$ covering $\gamma(e), \gamma(b)$, respectively, are a distance at most $C_{3}$ apart on segments of length at least $N \cdot \operatorname{length}(\gamma(b))$.

By convexity of distance in a hyperbolic space, for any $\epsilon>0$ there is a constant $C_{4}(\epsilon)$ such that these lifts are $\epsilon$-close on segments of length at least $N \cdot \operatorname{length}(\gamma(b))$ $C_{4}$. This tells us how to choose $N$. We choose $\epsilon$ such that $8 \epsilon$ is less than the length of the shortest nontrivial loop on $S$, and choose $N$ so that

$$
N \cdot \operatorname{length}(\gamma(b))-C_{4}>2 \cdot \operatorname{length}(\gamma(b))+4 \epsilon
$$

in order to be able to apply Lemma 3.2,

Note that length $(\gamma(b)) \geq 8 \epsilon$, and $\epsilon$ is bounded below by a positive constant depending only on the geometry of $S$, so $N, \epsilon$ as above depend only on $S$.

Let $\tau$ be a segment of $\tilde{\gamma}(e)$ coming from a copy of $\sigma$ in a realizing path for $c_{\sigma}$ on $e^{C_{1}}$, which is $\epsilon$-close to a segment of $\tilde{\gamma}(b)$. Since $\tau$ by hypothesis has length at least $2 \cdot \operatorname{length}(\gamma(b))+4 \epsilon$, by Lemma 3.2, the projection of $\tau$ to $S$ contributes at least one transverse self-intersection to $\gamma\left(e^{C_{1}}\right)$.

If a realizing path for $e^{C_{1}}$ contains $p$ disjoint copies of $\sigma$, we obtain $p$ such segments $\tau_{1}, \ldots, \tau_{p}$, and therefore at least $p^{2}$ self-intersections of $\gamma\left(e^{C_{1}}\right)$. Since $\operatorname{cr}\left(e^{C_{1}}\right)=C_{1}^{2} \operatorname{cr}(e) \leq C_{1}^{2} n$ we obtain an estimate $p^{2} \leq C_{1}^{2} n$. 
Obviously a similar estimate holds for copies of $\sigma^{-1}$ in $e^{C_{1}}$. Therefore we obtain

$$
\left|h_{\sigma}\left(e^{C_{1}}\right)\right| \leq p \leq C_{1} \sqrt{n} .
$$

Note that after homogenizing, we will obtain a similar estimate for $e$.

By Lemma 2.5, the defect of $h_{\sigma}$ depends only on $S$, so we homogenize $h_{\sigma}$ and get a homogeneous quasimorphism $\bar{h}_{\sigma}$ with defect depending only on $S$, and satisfying

$$
\bar{h}_{\sigma}\left(b^{m}\right)=\frac{m}{N}, \quad \bar{h}_{\sigma}(e) \leq C_{5} \sqrt{n}+C_{6}
$$

for any integer $m$ and any $e \in S_{n}$, where $N, C_{5}, C_{6}$ depend only on $S$.

By the defining property of quasimorphisms (that they are additive with bounded error), we get a bound of the form

$$
w_{n}\left(b^{m}\right) \geq \frac{C_{7} m}{\sqrt{n}+C_{8}}-C_{9}
$$

where all constants depend only on $S$. Since $w_{n}\left(a^{m C_{1}}\right)=w_{n}\left(b^{m}\right)$, and $a$ is an arbitrary element with $\operatorname{cr}(a)>0$, this proves Theorem A.

Remark 4.1. In view of the estimates in this section, one might be tempted to guess that the crossing number itself is an appropriate function with which to measure word length in $\pi_{1}(S)$. Nevertheless, one can construct examples of pairs of elements $a, b \in \pi_{1}(S)$ such that $\operatorname{cr}(a)=\operatorname{cr}(b)=0$, but $\operatorname{cr}(a b)$ is as large as desired. For example, let $\gamma(b)$ be a very long simple geodesic which is very close (in the Hausdorff topology) to a minimal geodesic lamination, and let $\gamma(a)$ cross a collection of $10^{100}$ almost parallel strands of $\gamma(b)$. Let $p \in \gamma(a) \cap \gamma(b)$ be a basepoint, in order to pin down the based homotopy classes of $a, b$. Then the conjugacy class of $a b$ contains a representative loop (not a geodesic) which consists of the geodesic $\gamma(a)$ followed by the geodesic $\gamma(b)$. Most of the self-intersections of the loop $a b$ are essential, and therefore the geodesic $\gamma(a b)$ also has at least $10^{100}$ transverse self-intersections.

\section{ACKNOWLEDGMENTS}

While writing this paper I was partially supported by NSF grant DMS 0405491. I would like to thank Benson Farb for posing the question which this paper addresses, Chris Leininger for the observation mentioned in Remark 3.3, and the anonymous referee. This research was carried out while I was at the Tokyo Institute of Technology, hosted by Sadayoshi Kojima. I thank TIT and Professor Kojima for their hospitality.

\section{REFERENCES}

1. C. Bavard, Longeur stable des commutateurs, L'Enseign. Math. 37 (1991), 109-150. MR.1115747 (92g:20051)

2. M. Bridson and A. Haefliger, Metric spaces of non-positive curvature, Grund. der Math. Wiss. 319, Springer-Verlag, Berlin, 1999. MR1744486 (2000k:53038)

3. R. Brooks, Some remarks on bounded cohomology, Riemann surfaces and related topics: Proceedings of the 1978 Stony Brook Conference (State Univ. New York, Stony Brook, N.Y., 1978), 53-63, Ann. of Math. Stud., 97, Princeton Univ. Press, Princeton, N.J., 1981. MR624804 (83a:57038)

4. D. Calegari, scl, monograph; draft available for download from the author's webpage

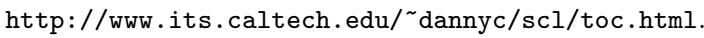

5. D. Calegari and K. Fujiwara, Stable commutator length in word-hyperbolic groups, preprint, math.GR/0611889. 
6. D. Epstein and K. Fujiwara, The second bounded cohomology of word-hyperbolic groups, Topology 36 (1997), 1275-1289. MR1452851 (98k:20088)

7. M. Gromov, Hyperbolic groups, in Essays in group theory, Math. Sci. Res. Inst. Publ., 8, Springer, New York, 1987. MR919829 (89e:20070)

Department of Mathematics, Caltech, Pasadena, California 91125

E-mail address: dannyc@its.caltech.edu 